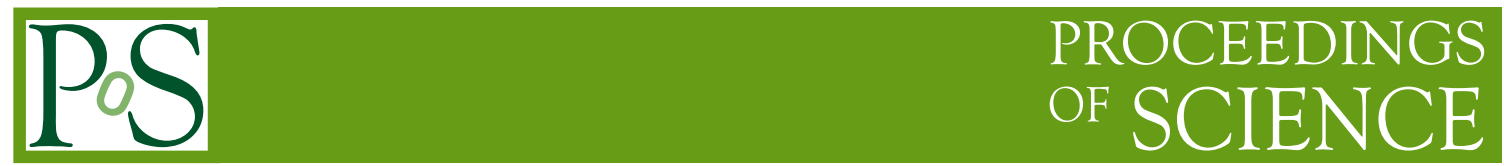

\title{
The Higgs Portal: Constraints on New Physics
}

\author{
Margarete Mühlleitner ${ }^{a, *}$ \\ ${ }^{a}$ Institute for Theoretical Physics, Karlsruhe Institute of Technology \\ Wolfgang-Gaede-Straße 1, 76128 Karlsruhe, Germany \\ E-mail: margarete.muehlleitner@kit.edu
}

In view of no direct sign of new physics so far and the pending puzzles that cannot be solved within the Standard Model (SM) the Higgs sector plays an increasingly important role as a portal to physics beyond the SM. New physics can be parametrized in a rather model-independent way in the SM effective theory (SMEFT). In this contribution I will discuss what we can learn about new physics from Higgs fits to SMEFT higher-dimensional operators.

The Eighth Annual Conference on Large Hadron Collider Physics-LHCP2020

25-30 May, 2020

online

${ }^{*}$ Speaker 


\section{Introduction}

The Higgs boson discovered in 2012 by the LHC experiments ATLAS [1] and CMS [2] behaves very Standard Model (SM)-like [3, 4]. Due to open questions that cannot be answered within the SM, however, one of the main goals of the LHC is to search for new physics (NP). Since we lack any direct discovery of physics beyond the SM (BSM) so far the Higgs sector as a portal to NP plays an important role in setting indirect limits on NP. In this contribution I will discuss how we can use Higgs fits to access NP and what we can learn from them.

On the theory side, NP can be described either in a model-dependent way within specific UV complete models like e.g. supersymmetry and the 2-Higgs-Doublet Model (2HDM) or also composite Higgs models as an example for a strongly interacting Higgs boson. Or the manifestations of BSM physics are described through anomalous couplings in a rather model-independent approach either by a linear (SMEFT) [5-11] or non-linear (EWChL) effective Lagrangian [12-17].

The SMEFT smoothly departs from the SM by assuming the SM field content and gauge symmetries and that NP only enters at some high energy scale $\Lambda$. The SM deviations are then described by higher-dimensional operators built from the SM fields. In this linear realization of the Higgs sector the Higgs boson is part of a doublet and the leading NP effects are described by dimension-6 (dim-6) operators that are suppressed by the NP scale $\Lambda^{2}$. The effective Lagrangian is hence given by ${ }^{1}$

$$
\mathcal{L}_{\text {eff }}=\mathcal{L}_{\mathrm{S} M}+\sum_{i} \frac{C_{i}^{(6)} O_{i}^{(6)}}{\Lambda^{2}}+O\left(\Lambda^{-4}\right)+\text { h.c. },
$$

where the sum extends on the possible dim-6 operators $O_{i}^{(6)}$ and their related Wilson coefficients $C_{i}^{(6)}$. In the non-linear realization on the other hand, the Higgs field can also be a singlet.

In the following sections I will discuss various aspects of SMEFT, starting with SMEFT at the top-Higgs interface in Sec. 2, followed by the status of a global SMEFT analysis in Sec. 3 and SMEFT in Higgs pair production in Sec. 4. I will finish in Sec. 5 with a brief discussion of NP aspects in specific UV-complete models, focussing on the Higgs pair production process.

\section{The Top-Higgs Interface}

The importance of the top quark in EFT fits derives from the fact that the top quark is the only fermion that couples with an order one Yukawa coupling to the Higgs boson so that it plays a privileged role in most BSM scenarios for electroweak symmetry breaking (EWSB) and the stabilisation of the weak scale. In weakly coupled models like supersymmetry (SUSY) this leads to the introduction of SUSY partners, the stops. Strongly coupled models on the other hand, where the Higgs boson is a composite object, come along with heavy top partners (in order to explain the fermion masses through partial compositeness [18-20]). Moreover, the precise knowledge of the top-Higgs interaction is crucial for the determination of the trilinear Higgs self-coupling from Higgs pair production.

The LHC processes at the top-Higgs interface that are particularly interesting for EFT analyses are besides top-quark pair production associated Higgs production with a top quark pair, inclusive

\footnotetext{
${ }^{1}$ A possible dim-5 operator for the generation of the neutrino masses is not relevant for LHC phenomenology.
} 


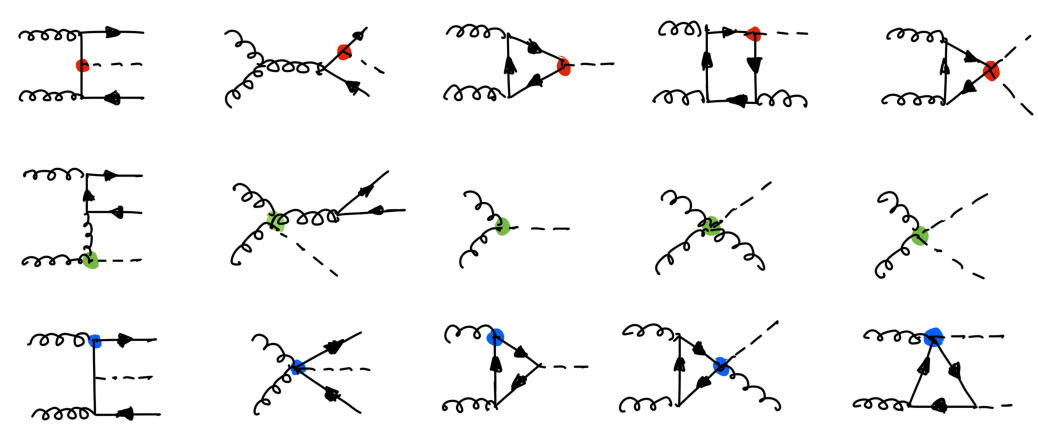

Figure 1: Sample diagrams contributing to associated top-Higgs production $t t H$ (two left columns), single Higgs production $H$ (third column), Higgs+jet production (fourth column) and Higgs pair production $H H$ (last column) together with the dim-6 operators involving the Higgs boson and the top quark, $O_{t \phi}(\mathrm{red}), O_{\phi G}$ (green) and $O_{t G}$ (blue).

Higgs production, Higgs+jet and Higgs pair production. The sample diagrams in Fig. 1 show which dim- 6 operators involving the Higgs boson and the top quark affect these processes. We have the operator $O_{t \phi}$ that modifies the top-Higgs interaction, the $O_{\phi G}$ operator inducing a point-like Higgs-gluon interaction and the chromomagnetic operator $O_{t G}$ modifying the interaction between tops and gluons,

$$
\begin{aligned}
O_{t \phi} & =\left(\phi^{\dagger} \phi\right)\left(\bar{Q}_{L} \tilde{\phi} t_{R}\right)+\text { h.c. } \\
O_{\phi G} & =\left(\phi^{\dagger} \phi\right) G_{\mu \nu}^{a} G^{a \mu \nu} \\
O_{t G} & =\left(\bar{Q}_{L} \sigma^{\mu \nu} T^{a} t_{R}\right) \phi G_{\mu \nu}^{a}+\text { h.c. },
\end{aligned}
$$

where $\phi$ denotes the Higgs doublet, $\tilde{\phi}=i \sigma^{2} H^{*}$ its charge conjugate, $Q$ the $S U(2)_{L}$ quark doublet built from the top and bottom quarks $t$ and $b, G_{\mu \nu}^{a}$ the gluon field strength tensor, $T^{a}$ the $S U$ (3) generators $(a=1, \ldots, 8)$, and $\sigma^{\mu \nu}=i / 2\left[\gamma^{\mu}, \gamma^{\nu}\right] .^{2}$

The description of inclusive Higgs production through effective operators [26-31] suffers from a two-fold degeneracy, namely in the operators $O_{t \phi}-O_{\phi G}$ and in $O_{t G}-O_{\phi G}$. The former degeneracy can be resolved by studying e.g. the transverse momentum distribution of single Higgs production [31-33]. As the operators $O_{t G}$ and $O_{\phi G}$ contribute to associated production of a Higgs boson with a top-quark pair in a different way, the remaining degeneracy between these two operators can be resolved by performing fits to their Wilson coefficients using input from single Higgs production, Higgs plus jet production [34-42] and $t \bar{t} H, c f$. Fig. 2 [43].

\section{Global Analysis}

An important aspect of the SMEFT analysis is that all relevant operators that contribute to the various processes included in a fit have to be used in order to keep it model- and basis-independent.

\footnotetext{
${ }^{2}$ Associated Higgs production with a top quark pair is also affected by four-fermion operators, which can be constrained from top-quark pair production [21] so that we do not consider them here further. The gluonic dim- 6 operator can be constrained from multijet-signatures [22] and is not discussed further here either. Finally, Higgs pair production is affected by the modification of the trilinear Higgs self-coupling [9, 23, 24] which will be investigated later in Sec. 4. CP-violating operators are neglected under the assumption of CP conservation [25].
} 

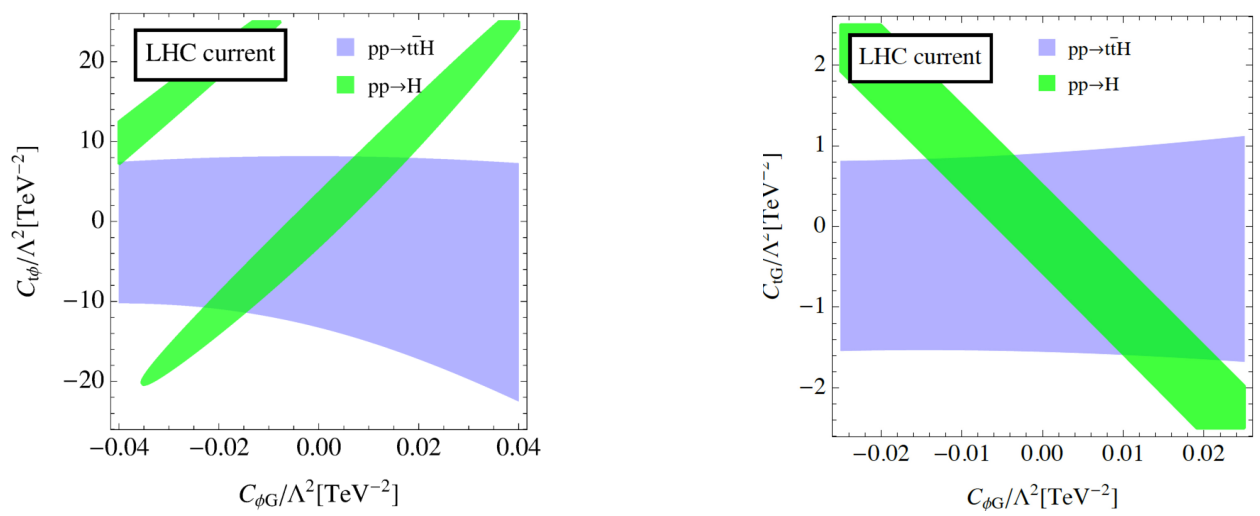

Figure 2: Allowed region in the $O_{t \phi}-O_{\phi G}$ plane (left) and the $O_{t G}-O_{\phi G}$ plane (right) at $95 \%$ confidence level. Plots taken from [43].

The problem is, however, that there is a large number of dim- 6 operators. For three fermion generations we have 2499 non-redundant operators and 59 for one generation [10]. A global SMEFT fit hence has to deal with a huge parameter space with possibly a large number of flat directions and local minima. A practical approach that is persued to reduce the number of operators in the fits is to apply symmetry assumptions like e.g. flavour and CP conservation and to focus on certain subsectors like e.g. Higgs, electroweak (EW), top, Higgs-EW, or Higgs-top. Then only the operators relevant for the considered particles and processes are included assuming that the remaining operators are well constrained from other processes. It has to be examined, however, to which extent this is justified in order not to draw wrong conclusions from the parameter fit.

Figure 3 shows an example of the 95\% CL limits in a fit including 34 degrees of freedom in the analysis, taking into account also next-to-leading order QCD corrections to various SMEFT contributions. For details and recent developments, see [45, 46]. The marginalised (global) and the individual fit cases are compared to the bounds from the LHC Top WG EFT note [44]. As can be inferred from the plot for almost all degrees of freedom the bounds obtained from the individual fits are comparable to or more stringent than the marginalised results from the global fit since in the former cross-correlations between the different directions spanned by the fitted degrees of freedom

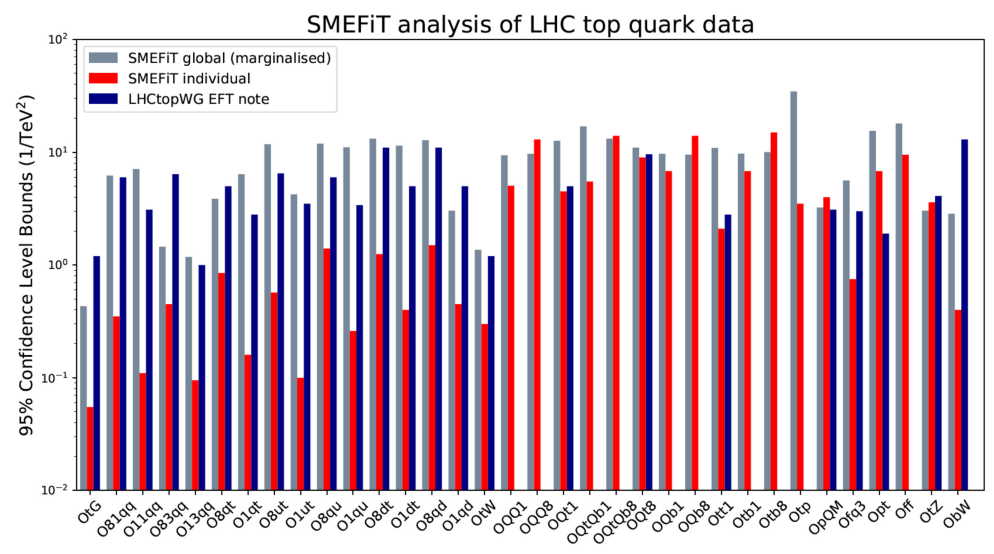

Figure 3: The 95\% CL bounds on 34 degrees of freedom in the marginalised (global) and the individual fit cases compared with the bounds from the LHC Top WG EFT note [44]. Plot taken from [45]. 

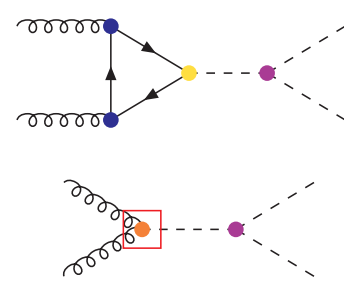
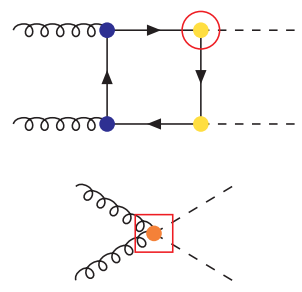

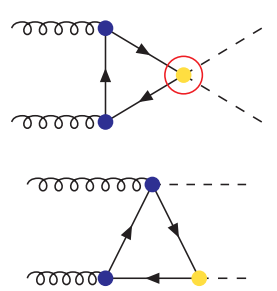

Figure 4: Dimension-6 operators affecting Higgs pair production: $O_{H}$ (not shown), $O_{6}$ (violet), $O_{t \phi}$ (yellow), $O_{\phi G}$ (orange), and $O_{t G}$ (blue). Boxes (circles) mark some examples of effective single- and double-Higgs couplings to gluons (fermions).

are neglected.

\section{Higgs Pair Production}

The measurement of the Higgs self-interactions provides the ultimate test of the Higgs mechanism [47]. The trilinear Higgs self-coupling is accessible in Higgs pair production with gluon fusion providing the dominant process at the LHC [48]. The dim-6 operators affecting Higgs pair production $[9,24,49]$ are shown in Fig. 4 . Besides an overall shift of the couplings due to $O_{H}$ the operators affect the Higgs self-coupling $\left(O_{6}\right)$ and the top Yukawa coupling $\left(O_{t \phi}\right)$. They furthermore induce effective single Higgs and double Higgs couplings to the gluons through $O_{\phi G}$, an effective 2-Higgs-2-fermion coupling through $O_{t \phi}$ and novel diagrams due to the chromomagnetic dipole operator $O_{t G}$. In the non-linear EFT approach the couplings between one and two Higgs bosons to the gluons (marked by the boxes) are not related as well as the single- and two-Higgs couplings to the fermions (markex by the circles), in contrast to the SMEFT approach at up to dimension 6 so that Higgs pair production becomes sensitive to the way EWSB is realized. When all other SMEFT parameters are fixed to their SM values, ATLAS (CMS) finds that the observed limits on the trilinear Higgs self-coupling at the 95\% CL lie between -5 and $+12(-11.8$ and 18.8) times the SM value $[50,51]$. With increasing precision on $H H$ production, however, the precise knowledge of the other Wilson coefficients will be required to bound the trilinear Higgs self-coupling.

An alternative way to constrain its values is given indirectly by exploiting single Higgs production where the trilinear Higgs self-coupling enters through EW corrections. ${ }^{3}$ It has been shown in several papers [54-59] that a competitive or at least complementary bound as compared to $H H$ production can be obtained. The challenge, however, is that several processes with different functional dependence on the coupling have to be considered which requires a global fit. Moreover, the dominant effect on single Higgs production comes from other leading-order operators (barring portal models where all SM couplings are unchanged but only the Higgs self-couplings are modified [56]). And finally, nine additional EFT coefficients have to be varied [58].

The potential of combining single and double Higgs fits to confine the trilinear coupling modifier $\kappa_{\lambda}$ [60] is shown in Fig. 5. It shows the constraints including differential observables in single and double Higgs production as well as their combination (pink). When including nonnegligible effects from all nine EFT parameters, double Higgs production leads to much stronger

\footnotetext{
${ }^{3}$ For limits on the trilinear Higgs self-coupling from two-loop effects in the EW observables, see [52, 53].
} 


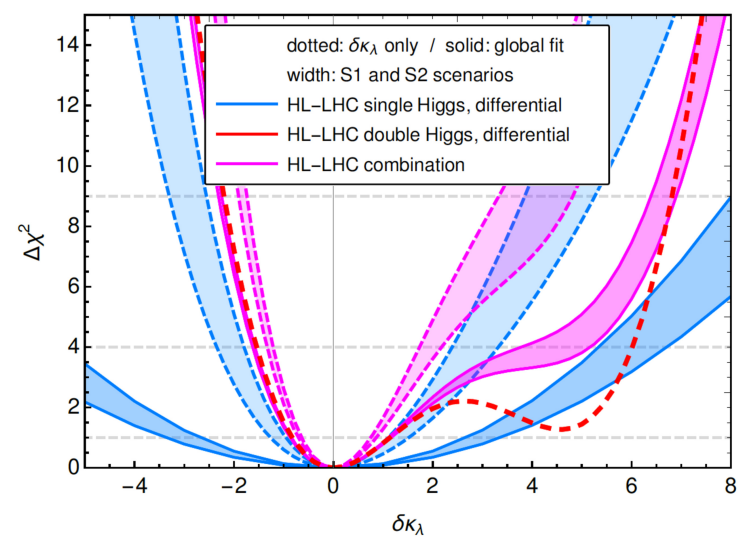

Figure 5: Constraints from differential single Higgs (blue), differential double Higgs (dashed red) production and their combination (pink) at the HL-LHC with $\sqrt{s}=13 \mathrm{TeV}$ and $\int \mathcal{L}=3 \mathrm{ab}^{-1}$. Taken from [60].

constraints. Single Higgs data are relevant, however, to lift the degenerate minima around $\delta \kappa_{\lambda} \sim 5$. The experiments have provided direct and indirect constraints on $\kappa_{\lambda}$ mostly by considering $\kappa_{\lambda}$ variations only. With increasing experimental sensitivity therefore the next steps to be performed are the simultaneous fit of both direct and indirect constraints and the inclusion of the relevant EFT operators in the analysis.

Distributions play a particularly important role in order to test NP and resolve degeneracies among the different EFT operators affecting the observable. The impact of the next-to-leading-order QCD corrections on the mass and $p_{T}$ distributions of Higgs pair production in the framework of a non-linearly realised EFT including the full mass dependence has been investigated in [61]. It is found that the mass effects lead to significant non-uniform $K$-factors as compared to the heavy top-limit [62].

\section{UV Complete Models}

In order to be sensitive to light resonances EFT analyses have to be complemented by investigations of specific UV complete models which shall be exemplified here for the case of Higgs pair production through gluon fusion. Here new physics can enter at several levels, as can be inferred from Fig. 6 showing sample diagrams contributing to Higgs pair production in the Next-to-MinimalSupersymmetric extension (NMSSM) (left) [63] and in the composite Higgs model (right) [64]. Thus the Higgs Yukawa couplings to the top and bottom quarks in the triangle and box diagrams as well as the trilinear Higgs self-coupling can be modified where in particular the deviation of the latter from its SM value can lead to larger cross sections [48]. Moreover, new particles can enter through the loops. Additional Higgs bosons $H_{k}$ can lead to a resonant enhancement of the cross section [23, 47, 63, 65]. In composite Higgs models additionally a novel 2-Higgs-2-fermion coupling appears. All these effects can induce cross sections that are considerably larger than the SM prediction [66].

Models with extended Higgs sectors can also lead to an interesting single-double top-Higgs interplay. Figure 7 shows the comparison of the invariant mass distribution for $p p \rightarrow t \bar{t}$ (left) and $p p \rightarrow h h$ (right) where $h$ denotes the SM-like among the three neutral CP-mixing Higgs bosons $H_{i}$ $(i=1,2,3)$ of the CP-violating 2HDM (C2HDM) [67-70], comparing the signal and interference 

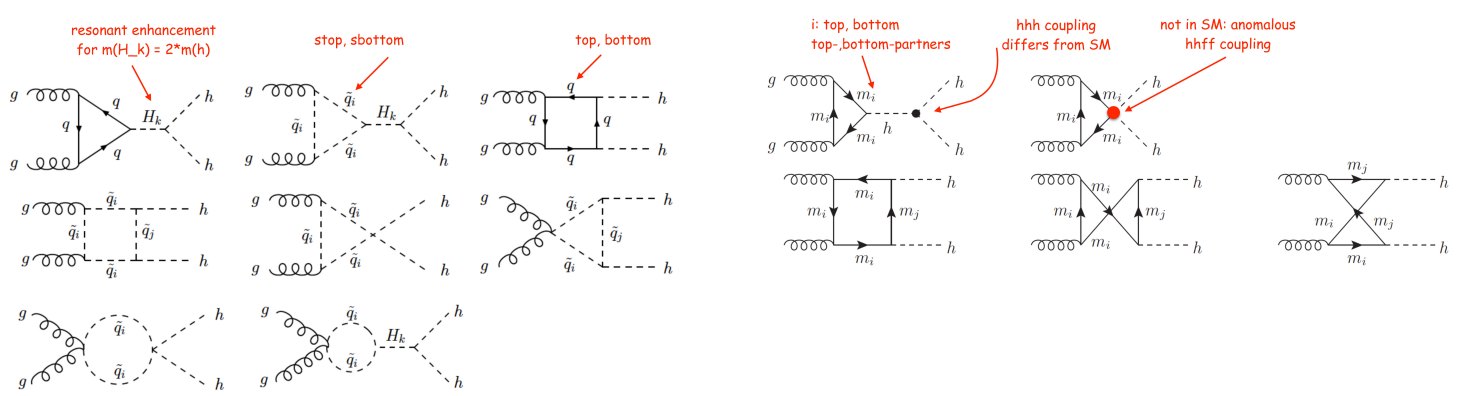

Figure 6: Sample diagrams contributing to Higgs pair production in the NMSSM (left) [63] and the composite Higgs model with top/bottom partners (right) [64].

cross sections. As can be inferred from these plots the destructive signal-background interference in the resonance search for heavy non-SM-like Higgs bosons renders single Higgs production in competition with the heavy Higgs discovery in Higgs pair production where constructive signalsional interferencec san anhanes the rroce certion 1711
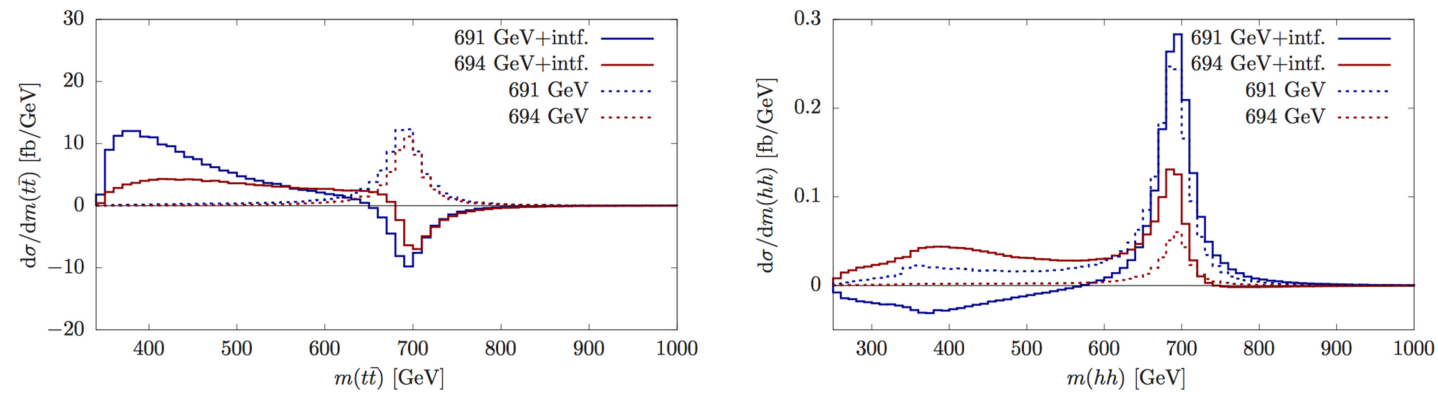

Figure 7: Invariant mass distribution for $p p \rightarrow t \bar{t}$ (left) and $p p \rightarrow h h$ (right) at $13 \mathrm{TeV}$ at leading order for the different Higgs states $H_{i} \neq h$ (blue: $H_{i}=H_{2}$, red: $H_{i}=H_{3}$ ). For details, see [71].

\section{Conclusions}

In this contribution, I discussed the SMEFT as a powerful framework to parametrize and test BSM physics in a rather model-independent way. We have seen that distributions play a particularly important role as they allow to test several NP operators at the same time and can resolve potential degeneracies. Global analyses taking into account all relevant observables are important to keep the analysis model- and basis-independent and they receive increasing attention. In the context of Higgs pair production the increasing experimental precision requires as next steps the simultaneous fit of both single and double Higgs processes and the gradual inclusion of all relevant EFT operators in the fits. The reality check with specific UV-complete models shows that in particular in Higgs pair production significant new physics effects are still possible.

\section{Acknowledgments}

I would like to thank the organizers of LHCP2020 for the invitation to this interesting workshop and the excellent organization of this online event on such a short time-scale. 


\section{References}

[1] ATLAS collaboration, Observation of a new particle in the search for the Standard Model Higgs boson with the ATLAS detector at the LHC, Phys. Lett. B 716 (2012) 1 [1207 . 7214].

[2] CMS collaboration, Observation of a New Boson at a Mass of $125 \mathrm{GeV}$ with the CMS Experiment at the LHC, Phys. Lett. B 716 (2012) 30 [1207.7235].

[3] ATLAS collaboration, Combined measurements of Higgs boson production and decay using up to $80 \mathrm{fb}^{-1}$ of proton-proton collision data at $\sqrt{\mathrm{s}}=13 \mathrm{TeV}$ collected with the ATLAS experiment, Phys. Rev. D 101 (2020) 012002 [1909. 02845].

[4] CMS collaboration, Combined measurements of Higgs boson couplings in proton-proton collisions at $\sqrt{s}=13 \mathrm{TeV}$, Eur. Phys. J. C 79 (2019) 421 [1809. 10733].

[5] C. Burges and H.J. Schnitzer, Virtual Effects of Excited Quarks as Probes of a Possible New Hadronic Mass Scale, Nucl. Phys. B 228 (1983) 464.

[6] C.N. Leung, S. Love and S. Rao, Low-Energy Manifestations of a New Interaction Scale: Operator Analysis, Z. Phys. C 31 (1986) 433.

[7] W. Buchmuller and D. Wyler, Effective Lagrangian Analysis of New Interactions and Flavor Conservation, Nucl. Phys. B 268 (1986) 621.

[8] K. Hagiwara, S. Ishihara, R. Szalapski and D. Zeppenfeld, Low-energy effects of new interactions in the electroweak boson sector, Phys. Rev. D 48 (1993) 2182.

[9] G. Giudice, C. Grojean, A. Pomarol and R. Rattazzi, The Strongly-Interacting Light Higgs, JHEP 06 (2007) 045 [hep-ph/0703164].

[10] B. Grzadkowski, M. Iskrzynski, M. Misiak and J. Rosiek, Dimension-Six Terms in the Standard Model Lagrangian, JHEP 10 (2010) 085 [1008 . 4884].

[11] J. Elias-Miro, J. Espinosa, E. Masso and A. Pomarol, Higgs windows to new physics through $d=6$ operators: constraints and one-loop anomalous dimensions, JHEP 11 (2013) 066 [1308. 1879].

[12] R. Contino, The Higgs as a Composite Nambu-Goldstone Boson, in Theoretical Advanced Study Institute in Elementary Particle Physics: Physics of the Large and the Small, pp. 235-306, 2011, DOI [1005 . 4269].

[13] G. Buchalla and O. Cata, Effective Theory of a Dynamically Broken Electroweak Standard Model at NLO, JHEP 07 (2012) 101 [1203.6510].

[14] R. Alonso, M. Gavela, L. Merlo, S. Rigolin and J. Yepes, The Effective Chiral Lagrangian for a Light Dynamical "Higgs Particle", Phys. Lett. B 722 (2013) 330 [1212 . 3305].

[15] G. Buchalla, O. Catà and C. Krause, Complete Electroweak Chiral Lagrangian with a Light Higgs at NLO, Nucl. Phys. B $\mathbf{8 8 0}$ (2014) 552 [1307. 5017]. 
[16] I. Brivio, T. Corbett, O. Éboli, M. Gavela, J. Gonzalez-Fraile, M. Gonzalez-Garcia et al., Disentangling a dynamical Higgs, JHEP 03 (2014) 024 [1311. 1823].

[17] G. Isidori, A.V. Manohar and M. Trott, Probing the nature of the Higgs-like Boson via $h \rightarrow V \mathcal{F}$ decays, Phys. Lett. B 728 (2014) 131 [1305.0663].

[18] D.B. Kaplan, Flavor at SSC energies: A New mechanism for dynamically generated fermion masses, Nucl. Phys. B 365 (1991) 259.

[19] R. Contino, T. Kramer, M. Son and R. Sundrum, Warped/composite phenomenology simplified, JHEP 05 (2007) 074 [hep-ph/0612180].

[20] M. Gillioz, R. Gröber, A. Kapuvari and M. Mühlleitner, Vector-like Bottom Quarks in Composite Higgs Models, JHEP 03 (2014) 037 [1311 . 4453].

[21] C. Zhang and S. Willenbrock, Effective-Field-Theory Approach to Top-Quark Production and Decay, Phys. Rev. D 83 (2011) 034006 [1008 . 3869].

[22] F. Krauss, S. Kuttimalai and T. Plehn, LHC multijet events as a probe for anomalous dimension-six gluon interactions, Phys. Rev. D 95 (2017) 035024 [1611.00767].

[23] S. Dawson, S. Dittmaier and M. Spira, Neutral Higgs boson pair production at hadron colliders: QCD corrections, Phys. Rev. D 58 (1998) 115012 [hep-ph/9805244].

[24] R. Contino, C. Grojean, M. Moretti, F. Piccinini and R. Rattazzi, Strong Double Higgs Production at the LHC, JHEP 05 (2010) 089 [1002 . 1011].

[25] R. Contino, M. Ghezzi, C. Grojean, M. Muhlleitner and M. Spira, Effective Lagrangian for a light Higgs-like scalar, JHEP 07 (2013) 035 [1303 . 3876].

[26] C. Degrande, J. Gerard, C. Grojean, F. Maltoni and G. Servant, Probing Top-Higgs Non-Standard Interactions at the LHC, JHEP 07 (2012) 036 [1205 . 1065].

[27] D. Choudhury and P. Saha, Higgs production as a probe of anomalous top couplings, JHEP 08 (2012) 144 [1201. 4130].

[28] G. Brooijmans et al., Les Houches 2015: Physics at TeV colliders - new physics working group report, in 9th Les Houches Workshop on Physics at TeV Colliders, 5, 2016 [1605.02684].

[29] R.V. Harlander, S. Liebler and H. Mantler, SusHi Bento: Beyond NNLO and the heavy-top limit, Comput. Phys. Commun. 212 (2017) 239 [1605. 03190].

[30] C. Anastasiou, C. Duhr, F. Dulat, E. Furlan, T. Gehrmann, F. Herzog et al., CP-even scalar boson production via gluon fusion at the LHC, JHEP 09 (2016) 037 [1605. 05761].

[31] N. Deutschmann, C. Duhr, F. Maltoni and E. Vryonidou, Gluon-fusion Higgs production in the Standard Model Effective Field Theory, JHEP 12 (2017) 063 [1708. 00460]. 
[32] M. Grazzini, A. Ilnicka, M. Spira and M. Wiesemann, Modeling BSM effects on the Higgs transverse-momentum spectrum in an EFT approach, JHEP 03 (2017) 115 [1612 . 00283].

[33] M. Grazzini, A. Ilnicka and M. Spira, Higgs boson production at large transverse momentum within the SMEFT: analytical results, Eur. Phys. J. C 78 (2018) 808 [1806. 08832].

[34] I. Low, R. Rattazzi and A. Vichi, Theoretical Constraints on the Higgs Effective Couplings, JHEP 04 (2010) 126 [0907 . 5413].

[35] C. Grojean, E. Salvioni, M. Schlaffer and A. Weiler, Very boosted Higgs in gluon fusion, JHEP 05 (2014) 022 [1312 . 3317].

[36] A. Azatov and A. Paul, Probing Higgs couplings with high $p_{T}$ Higgs production, JHEP 01 (2014) 014 [1309.5273].

[37] A. Banfi, A. Martin and V. Sanz, Probing top-partners in Higgs+jets, JHEP 08 (2014) 053 [1308.4771].

[38] M. Buschmann, D. Goncalves, S. Kuttimalai, M. Schonherr, F. Krauss and T. Plehn, Mass Effects in the Higgs-Gluon Coupling: Boosted vs Off-Shell Production, JHEP 02 (2015) 038 [1410.5806].

[39] S. Dawson, I. Lewis and M. Zeng, Effective field theory for Higgs boson plus jet production, Phys. Rev. D 90 (2014) 093007 [1409. 6299].

[40] M. Buschmann, C. Englert, D. Goncalves, T. Plehn and M. Spannowsky, Resolving the Higgs-Gluon Coupling with Jets, Phys. Rev. D 90 (2014) 013010 [1405.7651].

[41] M. Schlaffer, M. Spannowsky, M. Takeuchi, A. Weiler and C. Wymant, Boosted Higgs Shapes, Eur. Phys. J. C 74 (2014) 3120 [1405 . 4295].

[42] S. Dawson, I. Lewis and M. Zeng, Usefulness of effective field theory for boosted Higgs production, Phys. Rev. D 91 (2015) 074012 [1501.04103].

[43] F. Maltoni, E. Vryonidou and C. Zhang, Higgs production in association with a top-antitop pair in the Standard Model Effective Field Theory at NLO in QCD, JHEP 10 (2016) 123 [1607.05330].

[44] D. Barducci et al., Interpreting top-quark LHC measurements in the standard-model effective field theory, 1802.07237.

[45] N.P. Hartland, F. Maltoni, E.R. Nocera, J. Rojo, E. Slade, E. Vryonidou et al., A Monte Carlo global analysis of the Standard Model Effective Field Theory: the top quark sector, JHEP 04 (2019) 100 [1901.05965].

[46] C. Degrande, G. Durieux, F. Maltoni, K. Mimasu, E. Vryonidou and C. Zhang, Automated one-loop computations in the SMEFT, 2008.11743. 
[47] A. Djouadi, W. Kilian, M. Muhlleitner and P. Zerwas, Production of neutral Higgs boson pairs at LHC, Eur. Phys. J. C 10 (1999) 45 [hep-ph/9904287].

[48] J. Baglio, A. Djouadi, R. Gröber, M. Mühlleitner, J. Quevillon and M. Spira, The measurement of the Higgs self-coupling at the LHC: theoretical status, JHEP 04 (2013) 151 [1212.5581].

[49] F. Goertz, A. Papaefstathiou, L.L. Yang and J. Zurita, Higgs boson pair production in the $D=6$ extension of the SM, JHEP 04 (2015) 167 [1410 . 3471].

[50] CMS collaboration, Combination of searches for Higgs boson pair production in proton-proton collisions at $\sqrt{s}=13 \mathrm{TeV}$, Phys. Rev. Lett. 122 (2019) 121803 [1811.09689].

[51] ATLAS collaboration, Combination of searches for Higgs boson pairs in pp collisions at $\sqrt{s}=13 \mathrm{TeV}$ with the ATLAS detector, Phys. Lett. B 800 (2020) 135103 [1906.02025].

[52] G.D. Kribs, A. Maier, H. Rzehak, M. Spannowsky and P. Waite, Electroweak oblique parameters as a probe of the trilinear Higgs boson self-interaction, Phys. Rev. D 95 (2017) 093004 [1702.07678].

[53] G. Degrassi, M. Fedele and P.P. Giardino, Constraints on the trilinear Higgs self coupling from precision observables, JHEP 04 (2017) 155 [1702 . 01737].

[54] M. McCullough, An Indirect Model-Dependent Probe of the Higgs Self-Coupling, Phys. Rev. $D 90$ (2014) 015001 [1312.3322].

[55] M. Gorbahn and U. Haisch, Indirect probes of the trilinear Higgs coupling: $g g \rightarrow h$ and $h \rightarrow \gamma \gamma$, JHEP 10 (2016) 094 [1607.03773].

[56] G. Degrassi, P.P. Giardino, F. Maltoni and D. Pagani, Probing the Higgs self coupling via single Higgs production at the LHC, JHEP 12 (2016) 080 [1607.04251].

[57] W. Bizon, M. Gorbahn, U. Haisch and G. Zanderighi, Constraints on the trilinear Higgs coupling from vector boson fusion and associated Higgs production at the LHC, JHEP 07 (2017) 083 [1610.05771].

[58] S. Di Vita, C. Grojean, G. Panico, M. Riembau and T. Vantalon, A global view on the Higgs self-coupling, JHEP 09 (2017) 069 [1704.01953].

[59] M. Gorbahn and U. Haisch, Two-loop amplitudes for Higgs plus jet production involving a modified trilinear Higgs coupling, JHEP 04 (2019) 062 [1902 . 05480].

[60] J. Alison et al., Higgs Boson Pair Production at Colliders: Status and Perspectives, in Double Higgs Production at Colliders, B. Di Micco, M. Gouzevitch, J. Mazzitelli and C. Vernieri, eds., 9, 2019, DOI [1910.00012].

[61] G. Buchalla, M. Capozi, A. Celis, G. Heinrich and L. Scyboz, Higgs boson pair production in non-linear Effective Field Theory with full $m_{t}$-dependence at NLO QCD, JHEP 09 (2018) 057 [1806.05162]. 
[62] R. Grober, M. Muhlleitner, M. Spira and J. Streicher, NLO QCD Corrections to Higgs Pair Production including Dimension-6 Operators, JHEP 09 (2015) 092 [1504. 06577].

[63] D.T. Nhung, M. Muhlleitner, J. Streicher and K. Walz, Higher Order Corrections to the Trilinear Higgs Self-Couplings in the Real NMSSM, JHEP 11 (2013) 181 [1306. 3926].

[64] R. Grober, M. Muhlleitner and M. Spira, Signs of Composite Higgs Pair Production at Next-to-Leading Order, JHEP 06 (2016) 080 [1602.05851].

[65] R. Grober, M. Muhlleitner and M. Spira, Higgs Pair Production at NLO QCD for CP-violating Higgs Sectors, Nucl. Phys. B 925 (2017) 1 [1705 . 05314].

[66] P. Basler, S. Dawson, C. Englert and M. Mühlleitner, Showcasing HH production: Benchmarks for the LHC and HL-LHC, Phys. Rev. D 99 (2019) 055048 [1812 . 03542].

[67] G. Branco and M. Rebelo, The Higgs Mass in a Model With Two Scalar Doublets and Spontaneous \{CP\} Violation, Phys. Lett. B 160 (1985) 117.

[68] I.F. Ginzburg, M. Krawczyk and P. Osland, Two Higgs doublet models with CP violation, in International Workshop on Linear Colliders (LCWS 2002), pp. 703-706, 11, 2002 [hep-ph/0211371].

[69] W. Khater and P. Osland, CP violation in top quark production at the LHC and two Higgs doublet models, Nucl. Phys. B 661 (2003) 209 [hep-ph/0302004].

[70] D. Fontes, M. Mühlleitner, J.C. Romão, R. Santos, J.a.P. Silva and J. Wittbrodt, The C2HDM revisited, JHEP 02 (2018) 073 [1711.09419].

[71] P. Basler, S. Dawson, C. Englert and M. Mühlleitner, Di-Higgs boson peaks and top valleys: Interference effects in Higgs sector extensions, Phys. Rev. D 101 (2020) 015019 [1909.09987]. 